\title{
Application Of CPM Methode (Critical Path Methode) In Controlling the Time 100 Teus Contrainer Ship Hull Construction Project
}

\author{
Mia Syafrina, Fandy Bestario Harlan \\ * Batam Polytechnics \\ Department of Business Management \\ Parkway Street, Batam Centre, Batam 29461, Indonesia \\ E-mail: miasyafrina@polibatam.ac.id, fandybestario@polibatam.ac.id
}

\begin{abstract}
Abstrak
Proyek konstruksi umumnya merupakan usaha yang paling berisiko tinggi khususnya proyek pembangunan kapal. Upaya pengurangan resiko tersebut dapat dilakukan dengan meminimalkan potensi resiko. Penelitian ini bertujuan untuk melihat potensi resiko yang tinggi dan mencegah keterlambatan penyelesaian konstruksi kapal menggunakan Critial Path Method CPM pada PT.XYZ. Dengan menggunakan Critial Path Method CPM jalur - jalur kritis dapat diberikan perhatian lebih sehingga tidak akan mengganggu proyek konstruksi kapal. Selain itu juga menjadi bentuk antisipasi jika ada keterlambatan maka memungkinkan untuk dilakukan penjadwalan kembali.
\end{abstract}

Kata kunci: Metode CPM, Jalur - Jalur Kritis, Konstruksi Kapal

\begin{abstract}
Construction projects are generally the most high-risk businesses, especially shipbuilding projects. Efforts to reduce the risk can be done by minimizing the potential risk. This study aims to see potential high risk and prevent delays in the completion of ship construction using the Critical Path Method CPM at PT. XYZ. By using the Critical Path Method CPM critical paths can be given more attention so that they will not interfere ship construction projects. In addition, it is also a form of anticipation if there is a delay, it is possible to reschedule.
\end{abstract}

Keywords: CPM Methode, Critical Path, Shipbuilding

17 | Jurnal Akuntansi, Ekonomi dan Manajemen Bisnis | Vol. 9 No.1, July 2021, 17-24 | E-ISSN: 2548-9836 


\section{Introduction}

Construction projects are generally the most high-risk businesses [1]. The ship construction project is a competitive project with major risks such as the delay in delivery of the vessel to the owner, work accidents, and production errors that cause the ship not to match the owner's order so with a large level of risk need good planning and control in the field. Based on the Research Report on Shipbuilding Japan Cho et al (1996) it is known that the hull construction process is $48-50 \%$ of the shipbuilding process [2]. Delay is defined as the time overrun either beyond the contract's date or beyond the delivery date agreed upon by the project's different parties. PT. XYZ is a ship construction company collaborates with Damen Shipyard Gorinchem for the construction of new ships. In its planning, PT XYZ plans to build a 100 TEUS container ship on time and well managed. Using critical path analysis in ship construction scheduling planning is one of the most effective and efficient methods to prevent delays in ship construction completion.

\subsection{Ship Construction}

The stages of the hull construction itself are divided into several stages of work. The construction of the ship's hull can be divided into 4 stages [9], namely:

1. Fabrication Part: The fabrication is the earliest level of physical work when building a new ship. The fabrication section consists of several main activities, such as: marking, cutting, roll, press, bending

2. Sub-assembly Part: Sub-assembly process consists of fit-up and welding of production parts that have been carried out at the fabrication stage. Sub assembly process is combining several small components into components per panel.

3. Block assembly Part: Block assembly stage is one of core work on ship construction. Block assembly is the activity combining two or more panels to form a ship block. This merging process usually requires heavy equipment to support its production activities. This stage consists of 4 main activities, namely: JIG preparation, scantling check, fitup, inspection.

\subsection{Critical Path Method}

Critical Path Method (CPM) is a technique for analyzing projects by determining the longest sequence of tasks (or the sequence of task with the least slack) through a project network [5]. The function of the critical path is to find out activities that have a very high sensitivity to delays in completing work, or also called critical activities [9]. By concentrating on the most critical tasks it can be ensured that the project is on time and is keeping pace with the schedule set up [3]. The project network is a flow chart that graphically depicts the sequence, inter dependence of the project work plan. One of the functions of the project network is to analyze activities that are on a critical path.

\subsection{Project Management}

A project is a complex undertaking, nonroutine, one-time activity constrained by time, budget, resources, and performance specifications designed to meet customer requirements. The purpose of project management is that these activities can be achieved efficiently and effectively. In general, the project cycle and process from start to finish are as follows:

1. Defining

2. Planning

3. Executing

4. Closure

\subsection{Term in Project Networking}

Some of the terms often used in project networking are:

- ESij (early start), is earliest start time on activity $(\mathrm{i}, \mathrm{j})$ [4]. ES for each element $(i, j)$ is equal with Ei for previous element.

$$
S_{i j}=E_{i}
$$

- EF (early finish), is how fast an activity can be completed. EF for each element $(i, j)$ is equal with ES plus duration of event.

$$
E F_{i j}=E S_{i j}+D_{i j}
$$

- LF (late finish), is how late the activity can be completed. At each node we calculate the least finish and start energy for each activity by considering. $\mathrm{Lj}$ as the maximum/ latest occurrence

18 | Jurnal Akuntansi, Ekonomi dan Manajemen Bisnis | Vol. 9 No.1, July 2021, 17-24 | E-ISSN: 2548-9836 
of node $j[6]$. LF for each element $(i, j)$ is equal with LET from previous $\mathrm{j}$.

$$
L F_{i j}=L_{j}
$$

- LS (late start), is how late an activity can be started. LS fo each elemenat $(i, j)$ is equal with LF minus duration of event.

$$
L S_{i j}=L F_{i j}-D_{i j}
$$

- Slack / Float, is how long the activity is delayed. Activity $i$ is regarded as a critical activity if its float time is zero [7].

\subsection{Arranging of Critical Path Network}

1. List project / process activities

2. Draw a diagram

3. Calculate \& analyze the earliest event time (EET)

4. Calculate \& analyze the latest event time (LET)

5. Determine the critical path

\section{Methods}

The type of study is case study research. Case study research, through reports of past studies, allows the exploration and understanding of complex issues. It can be considered a robust research method particularly when a holistic, in-depth investigation is required.

The data needed in this study will be taken from production data construction of new hull construction. The primary data needed are: data on the intensity of the occurrence of each type of waste. Data collection the weight of the intensity of the waste is carried out by using a questionnaire method on several respondents in companies that have experience in their fields. Primary data the other is the respondent's data on the priority risk rating for the company.

The data will be processed to see the potential for high risk and prevent delays in the completion of ship construction using the Critical Path Method CPM.

\section{Results and Discussion}

\subsection{Data Collection}

Data collection was carried out at PT. XYZ with the object of research on the construction of a 100 TEUS container ship.

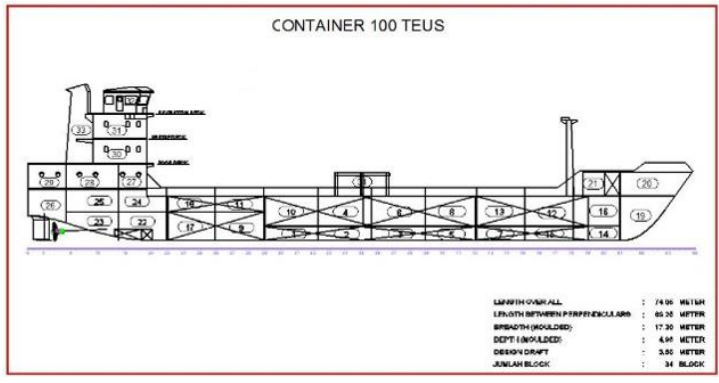

Figure 1. Container 100 TEUS Design Drawing

In the picture above, it can be seen that PT. XYZ builds 100 TEUS container ships with 33 blocks ship assembly part.

3.2 Compile a list of activities and the duration of their completion

The next step is to compile a list of activities and the timing of the activities. The number of activities can be seen based on the picture below, while the duration of activities is obtained from the project activity schedule.

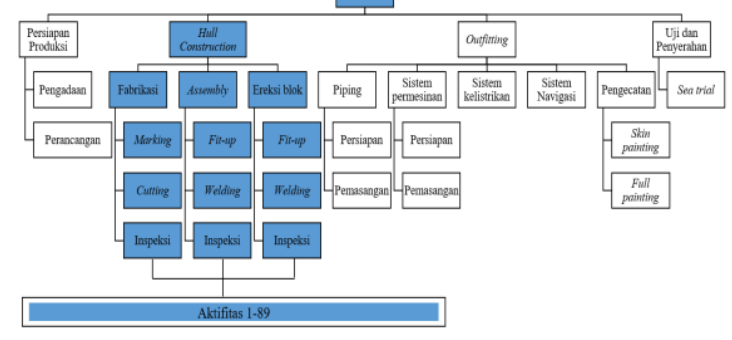

Figure 2. Ship Production Diagram

Then after determining the duration of the activity, perform a logical analysis of the dependence between activities. The logic of dependence between activities is obtained in the following way.

1. Determine the initial activity to be carried out, where the start of the activity does not depend on other activities.

2. Determine the next activity to be carried out when an activity has been completed.

3. Before starting an activity, what activity precedes the activity (dependency logic).

So that the preparation of the logic of dependence and duration of activities on the construction of a 100 TEUS container ship is as in table below: 
TABLE 1. DURATION OF EACH PROCESS

\begin{tabular}{|c|c|c|c|c|}
\hline No & Activity & Code & $\begin{array}{c}\text { Dependency } \\
\text { Logic }\end{array}$ & $\begin{array}{c}\text { Dura- } \\
\text { tion } \\
(\text { days }))\end{array}$ \\
\hline 1. & $\begin{array}{l}\text { Block Fabrica- } \\
\text { tion } 1\end{array}$ & A1 & Starting & 5 \\
\hline 2. & $\begin{array}{l}\text { Block Fabrica- } \\
\text { tion } 2\end{array}$ & $\mathrm{~A} 2$ & A1 & 5 \\
\hline 3. & $\begin{array}{l}\text { Block Assem- } \\
\text { bly1 }\end{array}$ & B1 & A1 & 26 \\
\hline 4. & $\begin{array}{l}\text { Block Assem- } \\
\text { bly2 }\end{array}$ & B2 & $\mathrm{A} 2$ & 30 \\
\hline 5. & $\begin{array}{l}\text { Block Fabrica- } \\
\text { tion } 3\end{array}$ & A3 & A2 & 5 \\
\hline 6. & $\begin{array}{l}\text { Block Assem- } \\
\text { bly3 }\end{array}$ & B3 & A3 & 28 \\
\hline 7. & $\begin{array}{l}\text { Block Fabrica- } \\
\text { tion } 5\end{array}$ & A4 & A3 & 5 \\
\hline 8. & $\begin{array}{l}\text { Block Assem- } \\
\text { bly5 }\end{array}$ & B4 & A4 & 25 \\
\hline 9. & $\begin{array}{l}\text { Block Fabrica- } \\
\text { tion } 7\end{array}$ & A5 & A4 & 5 \\
\hline 10. & $\begin{array}{l}\text { Block Assem- } \\
\text { bly7 }\end{array}$ & B5 & A5 & 25 \\
\hline 11. & $\begin{array}{l}\text { Block Fabrica- } \\
\text { tion } 4\end{array}$ & A6 & A5 & 8 \\
\hline 12. & $\begin{array}{l}\text { Block Assem- } \\
\text { bly4 }\end{array}$ & B6 & A6 & 25 \\
\hline 13. & $\begin{array}{l}\text { Block Fabrica- } \\
\text { tion } 6\end{array}$ & A7 & A6 & 5 \\
\hline 14. & $\begin{array}{l}\text { Block Assem- } \\
\text { bly6 }\end{array}$ & B7 & A7 & 29 \\
\hline 15. & $\begin{array}{l}\text { Block Fabrica- } \\
\text { tion } 10\end{array}$ & A8 & A7 & 5 \\
\hline 16. & $\begin{array}{l}\text { Block Assem- } \\
\text { bly10 }\end{array}$ & B8 & A8 & 32 \\
\hline 17. & $\begin{array}{l}\text { Erection Block } \\
1-2-3\end{array}$ & $\mathrm{C} 1$ & $\mathrm{~B} 1, \mathrm{~B} 2, \mathrm{~B} 3$ & 40 \\
\hline 18. & $\begin{array}{l}\text { Block Fabrica- } \\
\text { tion9 }\end{array}$ & A9 & A8 & 5 \\
\hline 19. & $\begin{array}{l}\text { Block Assem- } \\
\text { bly9 }\end{array}$ & B9 & A9 & 42 \\
\hline 20. & $\begin{array}{l}\text { Block Fabrica- } \\
\text { tion } 17\end{array}$ & A 10 & A9 & 5 \\
\hline 21. & $\begin{array}{l}\text { Block Assem- } \\
\text { bly17 }\end{array}$ & B10 & A10 & 45 \\
\hline 22. & $\begin{array}{l}\text { Block Fabrica- } \\
\text { tion } 11\end{array}$ & A11 & A10 & 5 \\
\hline 23. & $\begin{array}{l}\text { Block Assem- } \\
\text { bly11 }\end{array}$ & B11 & A11 & 30 \\
\hline 24. & $\begin{array}{l}\text { Block Fabrica- } \\
\text { tion } 18\end{array}$ & A12 & A11 & 5 \\
\hline 25. & $\begin{array}{l}\text { Block Assem- } \\
\text { bly18 }\end{array}$ & B12 & A12 & 46 \\
\hline 26. & $\begin{array}{l}\text { Block Fabrica- } \\
\text { tion } 13\end{array}$ & A13 & A12 & 5 \\
\hline 27. & $\begin{array}{l}\text { Block Assem- } \\
\text { bly13 }\end{array}$ & B13 & A13 & 45 \\
\hline 28. & $\begin{array}{l}\text { Block Fabrica- } \\
\text { tion8 }\end{array}$ & A14 & A13 & 5 \\
\hline 29. & $\begin{array}{l}\text { Block Assem- } \\
\text { bly8 }\end{array}$ & B14 & A14 & 29 \\
\hline 30. & $\begin{array}{l}\text { Block Fabrica- } \\
\text { tion } 12\end{array}$ & A15 & A14 & 5 \\
\hline 31. & $\begin{array}{l}\text { Block Assem- } \\
\text { bly12 }\end{array}$ & B15 & A15 & 31 \\
\hline 32. & $\begin{array}{l}\text { Block Fabrica- } \\
\text { tion15 }\end{array}$ & A16 & A15 & 5 \\
\hline
\end{tabular}

\begin{tabular}{|c|c|c|c|c|}
\hline 33. & $\begin{array}{l}\text { Block Assem- } \\
\text { bly15 }\end{array}$ & B16 & A16 & 50 \\
\hline 34. & $\begin{array}{l}\text { Block Fabrica- } \\
\text { tion16 }\end{array}$ & A17 & A16 & 5 \\
\hline 35. & $\begin{array}{l}\text { Block Assem- } \\
\text { bly16 }\end{array}$ & B17 & A17 & 50 \\
\hline 36. & $\begin{array}{l}\text { Block Fabrica- } \\
\text { tion14 }\end{array}$ & A18 & A17 & 5 \\
\hline 37. & $\begin{array}{l}\text { Block Assem- } \\
\text { bly14 }\end{array}$ & B18 & A18 & 40 \\
\hline 38. & $\begin{array}{l}\text { Erection Block } \\
5-7\end{array}$ & $\mathrm{C} 2$ & $\mathrm{~B} 4, \mathrm{~B} 5$ & 46 \\
\hline 39. & $\begin{array}{l}\text { Block Fabrica- } \\
\text { tion19 }\end{array}$ & A19 & A18 & 5 \\
\hline 40. & $\begin{array}{l}\text { Block Assem- } \\
\text { bly19 }\end{array}$ & B19 & A19 & 55 \\
\hline 41. & $\begin{array}{l}\text { Block Fabrica- } \\
\text { tion20 }\end{array}$ & A20 & A19 & 5 \\
\hline 42. & $\begin{array}{l}\text { Block Assem- } \\
\text { bly20 }\end{array}$ & B20 & A20 & 53 \\
\hline 43. & $\begin{array}{l}\text { Erection Block } \\
9-17\end{array}$ & C3 & B9, B10 & 43 \\
\hline 44. & $\begin{array}{l}\text { Block Fabrica- } \\
\text { tion21 }\end{array}$ & A21 & A20 & 5 \\
\hline 45. & $\begin{array}{l}\text { Block Assem- } \\
\text { bly21 }\end{array}$ & B21 & A21 & 58 \\
\hline 46. & $\begin{array}{l}\text { Erection Block } \\
9-10\end{array}$ & $\mathrm{C} 4$ & $\mathrm{C} 1, \mathrm{~B} 8, \mathrm{~B} 9$ & 25 \\
\hline 47. & $\begin{array}{l}\text { Block Fabrica- } \\
\text { tion22 }\end{array}$ & A22 & A21 & 5 \\
\hline 48. & $\begin{array}{l}\text { Block Assem- } \\
\text { bly22 }\end{array}$ & B22 & A22 & 52 \\
\hline 49. & $\begin{array}{l}\text { Block Fabrica- } \\
\text { tion23 }\end{array}$ & A23 & A22 & 5 \\
\hline 50. & $\begin{array}{l}\text { Block Assem- } \\
\text { bly23 }\end{array}$ & B23 & A23 & 60 \\
\hline 51. & $\begin{array}{l}\text { Block Fabrica- } \\
\text { tion24 }\end{array}$ & A24 & A23 & 5 \\
\hline 52. & $\begin{array}{l}\text { Block Assem- } \\
\text { bly24 }\end{array}$ & B24 & A24 & 49 \\
\hline 53. & $\begin{array}{l}\text { Block Fabrica- } \\
\text { tion25 }\end{array}$ & A25 & A24 & 5 \\
\hline 54. & $\begin{array}{l}\text { Block Assem- } \\
\text { bly25 }\end{array}$ & B25 & A25 & 66 \\
\hline 55. & $\begin{array}{l}\text { Block Fabrica- } \\
\text { tion26 }\end{array}$ & A26 & A25 & 5 \\
\hline 56. & $\begin{array}{l}\text { Block Assem- } \\
\text { bly26 }\end{array}$ & B26 & A26 & 70 \\
\hline 57. & $\begin{array}{l}\text { Block Fabrica- } \\
\text { tion27 }\end{array}$ & A27 & A26 & 5 \\
\hline 58. & $\begin{array}{l}\text { Block Assem- } \\
\text { bly27 }\end{array}$ & B27 & A27 & 57 \\
\hline 59. & $\begin{array}{l}\text { Erection Block } \\
4-6\end{array}$ & C5 & C1, B6, B7 & 32 \\
\hline 60. & $\begin{array}{l}\text { Block Fabrica- } \\
\text { tion } 28\end{array}$ & A28 & A27 & 5 \\
\hline 61. & $\begin{array}{l}\text { Block Assem- } \\
\text { bly28 }\end{array}$ & B28 & A28 & 67 \\
\hline 62. & $\begin{array}{l}\text { Block Fabrica- } \\
\text { tion29 }\end{array}$ & A29 & A28 & 5 \\
\hline 63. & $\begin{array}{l}\text { Block Assem- } \\
\text { bly29 }\end{array}$ & B29 & A29 & 65 \\
\hline 64. & $\begin{array}{l}\text { Erection Block } \\
11-18\end{array}$ & C6 & $\mathrm{B} 11, \mathrm{~B} 12, \mathrm{C} 3$ & 56 \\
\hline 65. & $\begin{array}{l}\text { Block Fabrica- } \\
\text { tion30 }\end{array}$ & A30 & A29 & 5 \\
\hline 66. & $\begin{array}{l}\text { Block Assem- } \\
\text { bly30 }\end{array}$ & $\mathrm{B} 30$ & A30 & 72 \\
\hline
\end{tabular}

20 | Jurnal Akuntansi, Ekonomi dan Manajemen Bisnis | Vol. 9 No.1, July 2021, 17-24 | E-ISSN: 2548-9836 


\begin{tabular}{|r|l|c|c|c|}
\hline 67. & $\begin{array}{l}\text { Block Fabrica- } \\
\text { tion31 }\end{array}$ & A31 & A30 & 5 \\
\hline 68. & $\begin{array}{l}\text { Block Assem- } \\
\text { bly31 }\end{array}$ & B31 & A31 & 75 \\
\hline 69. & $\begin{array}{l}\text { Block Fabrica- } \\
\text { tion32 }\end{array}$ & A32 & A31 & 5 \\
\hline 70. & $\begin{array}{l}\text { Block Assem- } \\
\text { bly32 }\end{array}$ & B32 & A32 & 70 \\
\hline 71. & $\begin{array}{l}\text { Block Fabrica- } \\
\text { tion33 }\end{array}$ & A33 & A32 & 5 \\
\hline 72. & $\begin{array}{l}\text { Block Assem- } \\
\text { bly33 }\end{array}$ & B33 & A33 & 48 \\
\hline 73. & $\begin{array}{l}\text { Erection Block } \\
8-13\end{array}$ & $\mathrm{C} 7$ & B14, B13, C2 & 50 \\
\hline 74. & $\begin{array}{l}\text { Block Fabrica- } \\
\text { tion34 }\end{array}$ & A34 & A33 & 5 \\
\hline 75. & $\begin{array}{l}\text { Block Assem- } \\
\text { bly34 }\end{array}$ & B34 & A34 & 36 \\
\hline 76. & $\begin{array}{l}\text { Erection Block } \\
12-15\end{array}$ & C8 & B16, B15 & 45 \\
\hline 77. & $\begin{array}{l}\text { Erection Block } \\
22-23\end{array}$ & C9 & B22, B23 & 63 \\
\hline 78. & $\begin{array}{l}\text { Erection Block } \\
24-25\end{array}$ & C10 & B24, B25, C9 & 66 \\
\hline
\end{tabular}

Basically, this critical chain calculation aims to determine the critical activities in the overall project activity. The way to determine critical activities in critical chain analysis is to calculate the float time available for activities. Float time is waiting time for

\begin{tabular}{|c|c|c|c|c|}
\hline 79. & $\begin{array}{l}\text { Erection Block } \\
26\end{array}$ & C11 & B26 & 68 \\
\hline 80. & $\begin{array}{l}\text { Erection Block } \\
27-28-29\end{array}$ & $\mathrm{C} 12$ & $\begin{array}{c}\text { B27, B28, B29, } \\
\text { C10, C11 }\end{array}$ & 71 \\
\hline 81. & $\begin{array}{l}\text { Erection Block } \\
30\end{array}$ & $\mathrm{C} 13$ & $\mathrm{~B} 30, \mathrm{C} 12$ & 60 \\
\hline 82. & $\begin{array}{l}\text { Erection Block } \\
14-16\end{array}$ & C14 & B17, B18 & 50 \\
\hline 83. & $\begin{array}{l}\text { Erection Block } \\
19\end{array}$ & $\mathrm{C} 15$ & B19 & 55 \\
\hline 84. & $\begin{array}{l}\text { Erection Block } \\
20-21\end{array}$ & C16 & $\begin{array}{c}\text { B20, B21, C14, } \\
\text { C15 }\end{array}$ & 50 \\
\hline 85. & $\begin{array}{l}\text { Erection Block } \\
34\end{array}$ & $\mathrm{C} 17$ & $\mathrm{~B} 34, \mathrm{C} 5$ & 28 \\
\hline 86. & $\begin{array}{l}\text { Erection Block } \\
31\end{array}$ & $\mathrm{C} 18$ & B31, C13 & 60 \\
\hline 87. & $\begin{array}{l}\text { Erection Block } \\
32\end{array}$ & C19 & B32, C18 & 57 \\
\hline 88. & $\begin{array}{l}\text { Erection Block } \\
33\end{array}$ & $\mathrm{C} 20$ & $\mathrm{~B} 33, \mathrm{C} 12$ & 41 \\
\hline 89. & $\begin{array}{l}\text { Launch prepa- } \\
\text { ration }\end{array}$ & $\mathrm{C} 21$ & Finish & \\
\hline
\end{tabular}

an activity before moving on to the next activity. The float time calculation is according to the formula in sub-chapter 2. In the construction of a 100 TEUS container ship, the critical activities for each of the entire project series are described in table below.

TABLE 2. CRITICAL ACTIVITY

\begin{tabular}{|c|c|c|c|c|c|c|c|c|}
\hline \multirow{2}{*}{$\begin{array}{l}\text { Activity } \\
\text { Code }\end{array}$} & \multirow{2}{*}{$\begin{array}{c}\text { Dependency } \\
\text { Logic }\end{array}$} & \multirow{2}{*}{$\begin{array}{l}\text { Duration } \\
\text { (days) }\end{array}$} & \multicolumn{5}{|c|}{ Time (Days) } & \multirow[t]{2}{*}{ Information } \\
\hline & & & EST & EFT & LST & LFT & Float & \\
\hline A1 & Start & 5 & 0 & 5 & 0 & 5 & 0 & Critical \\
\hline $\mathrm{A} 2$ & A1 & 5 & 5 & 10 & 5 & 10 & 0 & Critical \\
\hline B1 & A1 & 26 & 5 & 31 & 457 & 483 & 452 & \\
\hline B2 & A2 & 30 & 10 & 40 & 453 & 483 & 443 & \\
\hline A3 & A2 & 5 & 10 & 15 & 10 & 15 & 0 & Critical \\
\hline B3 & A3 & 28 & 15 & 43 & 455 & 483 & 440 & \\
\hline A4 & A3 & 5 & 15 & 20 & 15 & 20 & 0 & Critical \\
\hline B4 & A4 & 25 & 20 & 45 & 434 & 459 & 414 & \\
\hline A5 & A4 & 5 & 20 & 25 & 20 & 25 & 0 & Critical \\
\hline B5 & A5 & 25 & 25 & 50 & 434 & 459 & 409 & \\
\hline A6 & A5 & 8 & 25 & 33 & 25 & 33 & 0 & Critical \\
\hline B6 & A6 & 25 & 33 & 58 & 498 & 523 & 465 & \\
\hline A7 & A6 & 5 & 33 & 38 & 33 & 38 & 0 & Critical \\
\hline B7 & A7 & 29 & 38 & 67 & 494 & 523 & 456 & \\
\hline A8 & A7 & 5 & 38 & 43 & 38 & 43 & 0 & Critical \\
\hline B8 & A8 & 32 & 43 & 75 & 498 & 530 & 455 & \\
\hline $\mathrm{C} 1$ & $\mathrm{~B} 1, \mathrm{~B} 2, \mathrm{~B} 3$ & 40 & 43 & 83 & 483 & 523 & 440 & \\
\hline A9 & A8 & 5 & 38 & 43 & 38 & 43 & 0 & Critical \\
\hline B9 & A9 & 42 & 48 & 90 & 432 & 474 & 384 & \\
\hline A10 & A9 & 5 & 48 & 53 & 48 & 53 & 0 & Critical \\
\hline B10 & A10 & 45 & 53 & 98 & 429 & 474 & 376 & \\
\hline A11 & A10 & 5 & 53 & 58 & 53 & 58 & 0 & Critical \\
\hline B11 & A11 & 30 & 58 & 88 & 469 & 499 & 411 & \\
\hline A12 & A11 & 5 & 58 & 63 & 58 & 63 & 0 & Critical \\
\hline B12 & A12 & 46 & 63 & 109 & 453 & 499 & 390 & \\
\hline A13 & A12 & 5 & 63 & 68 & 63 & 68 & 0 & Critical \\
\hline B13 & A13 & 45 & 68 & 113 & 460 & 505 & 392 & \\
\hline A14 & A13 & 5 & 68 & 73 & 68 & 73 & 0 & Critical \\
\hline B14 & A14 & 29 & 73 & 102 & 476 & 505 & 403 & \\
\hline A15 & A14 & 5 & 73 & 78 & 73 & 78 & 0 & Critical \\
\hline B15 & A15 & 31 & 78 & 109 & 524 & 555 & 446 & \\
\hline A16 & A15 & 5 & 78 & 83 & 78 & 83 & 0 & Critical \\
\hline B16 & A16 & 50 & 83 & 133 & 505 & 555 & 422 & \\
\hline
\end{tabular}

21 | Jurnal Akuntansi, Ekonomi dan Manajemen Bisnis | Vol. 9 No.1, July 2021, 17-24 | E-ISSN: 2548-9836 


\begin{tabular}{|c|c|c|c|c|c|c|c|c|}
\hline A17 & A16 & 5 & 83 & 88 & 83 & 88 & 0 & Critical \\
\hline B17 & A17 & 50 & 88 & 138 & 108 & 158 & 20 & \\
\hline A18 & A17 & 5 & 88 & 93 & 88 & 93 & 0 & Critical \\
\hline B18 & A18 & 40 & 93 & 133 & 118 & 158 & 25 & \\
\hline $\mathrm{C} 2$ & B4, B5 & 46 & 50 & 96 & 459 & 505 & 409 & \\
\hline A19 & A18 & 5 & 93 & 98 & 93 & 98 & 0 & Critical \\
\hline B19 & A19 & 55 & 98 & 153 & 395 & 450 & 297 & \\
\hline A20 & A19 & 5 & 98 & 103 & 98 & 103 & 0 & Critical \\
\hline $\mathrm{B} 20$ & $\mathrm{~A} 20$ & 53 & 103 & 156 & 452 & 505 & 349 & \\
\hline $\mathrm{C} 3$ & B9, B10 & 43 & 98 & 123 & 474 & 499 & 376 & \\
\hline A21 & $\mathrm{A} 20$ & 5 & 103 & 108 & 103 & 108 & 0 & Critical \\
\hline B21 & A21 & 58 & 108 & 166 & 447 & 505 & 339 & \\
\hline $\mathrm{C} 4$ & $\mathrm{C} 1, \mathrm{~B} 8, \mathrm{~B} 9$ & 25 & 90 & 115 & 530 & 555 & 440 & \\
\hline A22 & $\mathrm{A} 21$ & 5 & 108 & 113 & 108 & 113 & 0 & Critical \\
\hline B22 & A22 & 52 & 113 & 165 & 126 & 178 & 13 & \\
\hline A23 & A22 & 5 & 113 & 118 & 113 & 118 & 0 & Critical \\
\hline B23 & A23 & 60 & 118 & 178 & 118 & 178 & 0 & Critical \\
\hline A24 & $\mathrm{A} 23$ & 5 & 118 & 123 & 154 & 159 & 36 & \\
\hline B24 & A24 & 49 & 123 & 172 & 192 & 241 & 69 & \\
\hline A25 & A24 & 5 & 123 & 128 & 159 & 164 & 36 & \\
\hline $\mathrm{B} 25$ & $\mathrm{~A} 25$ & 66 & 128 & 194 & 175 & 241 & 47 & \\
\hline A26 & A25 & 5 & 128 & 133 & 164 & 169 & 36 & \\
\hline B26 & A26 & 70 & 133 & 203 & 169 & 239 & 36 & \\
\hline A27 & A26 & 5 & 133 & 138 & 227 & 232 & 94 & \\
\hline B27 & A27 & 57 & 138 & 195 & 250 & 307 & 162 & \\
\hline $\mathrm{C} 5$ & C1, B6, B7 & 32 & 67 & 99 & 523 & 555 & 456 & \\
\hline A28 & A27 & 5 & 138 & 143 & 232 & 237 & 94 & \\
\hline B28 & A28 & 67 & 143 & 210 & 240 & 307 & 97 & \\
\hline A29 & A28 & 5 & 143 & 148 & 237 & 242 & 94 & \\
\hline B29 & A29 & 65 & 148 & 213 & 242 & 307 & 94 & \\
\hline C6 & $\mathrm{B} 11, \mathrm{~B} 12, \mathrm{C} 3$ & 56 & 123 & 179 & 499 & 555 & 376 & \\
\hline A30 & A29 & 5 & 148 & 153 & 301 & 306 & 153 & \\
\hline B30 & A30 & 72 & 153 & 225 & 306 & 378 & 153 & \\
\hline A31 & A30 & 5 & 153 & 158 & 358 & 363 & 205 & \\
\hline B31 & A31 & 75 & 158 & 233 & 363 & 438 & 205 & \\
\hline A32 & A31 & 5 & 158 & 163 & 376 & 381 & 218 & \\
\hline B32 & A32 & 70 & 163 & 233 & 428 & 498 & 265 & \\
\hline A33 & A32 & 5 & 163 & 168 & 381 & 386 & 218 & \\
\hline B33 & A33 & 48 & 168 & 216 & 466 & 514 & 298 & \\
\hline $\mathrm{C} 7$ & $\mathrm{~B} 14, \mathrm{~B} 13, \mathrm{C} 2$ & 50 & 113 & 173 & 505 & 555 & 382 & \\
\hline A34 & A33 & 5 & 168 & 173 & 386 & 491 & 318 & \\
\hline B34 & A34 & 36 & 173 & 209 & 491 & 527 & 318 & \\
\hline $\mathrm{C} 8$ & B16, B15 & 45 & 133 & 178 & 510 & 555 & 377 & \\
\hline C9 & $\mathrm{B} 22, \mathrm{~B} 23$ & 63 & 178 & 241 & 178 & 241 & 0 & Critical \\
\hline $\mathrm{C} 10$ & B24, B25, C9 & 66 & 241 & 307 & 241 & 307 & 0 & Critical \\
\hline C11 & B26 & 68 & 203 & 271 & 239 & 307 & 36 & \\
\hline $\mathrm{C} 12$ & $\begin{array}{c}\mathrm{B} 27, \mathrm{~B} 28, \mathrm{~B} 29, \\
\mathrm{C} 10, \mathrm{C} 11\end{array}$ & 71 & 307 & 378 & 307 & 378 & 0 & Critical \\
\hline C13 & B30, C12 & 60 & 378 & 438 & 378 & 438 & 0 & Critical \\
\hline $\mathrm{C} 14$ & B17, B18 & 50 & 138 & 188 & 158 & 208 & 20 & \\
\hline $\mathrm{C} 15$ & B19 & 55 & 153 & 208 & 450 & 505 & 297 & \\
\hline $\mathrm{C} 16$ & $\begin{array}{c}\text { B20, B21, C14, } \\
\text { C15 }\end{array}$ & 50 & 208 & 258 & 505 & 555 & 297 & \\
\hline $\mathrm{C} 17$ & B34, C5 & 28 & 209 & 237 & 527 & 555 & 318 & \\
\hline C18 & B31, C13 & 60 & 438 & 498 & 438 & 498 & 0 & Critical \\
\hline C19 & B32, C18 & 57 & 498 & 555 & 498 & 555 & 0 & Critical \\
\hline $\mathrm{C} 20$ & B33, C12 & 41 & 378 & 419 & 514 & 555 & 136 & \\
\hline $\mathrm{C} 21$ & Finish & 555 & & & & & & \\
\hline
\end{tabular}

In the table above, it can be seen that critical activities are activities that do not have waiting time for the next activity. So that critical activities are activity with zero float value. In addition, the critical path can also be seen from the project network diagram shown in fig 3 below.

22 | Jurnal Akuntansi, Ekonomi dan Manajemen Bisnis | Vol. 9 No.1, July 2021, 17-24 | E-ISSN: 2548-9836 


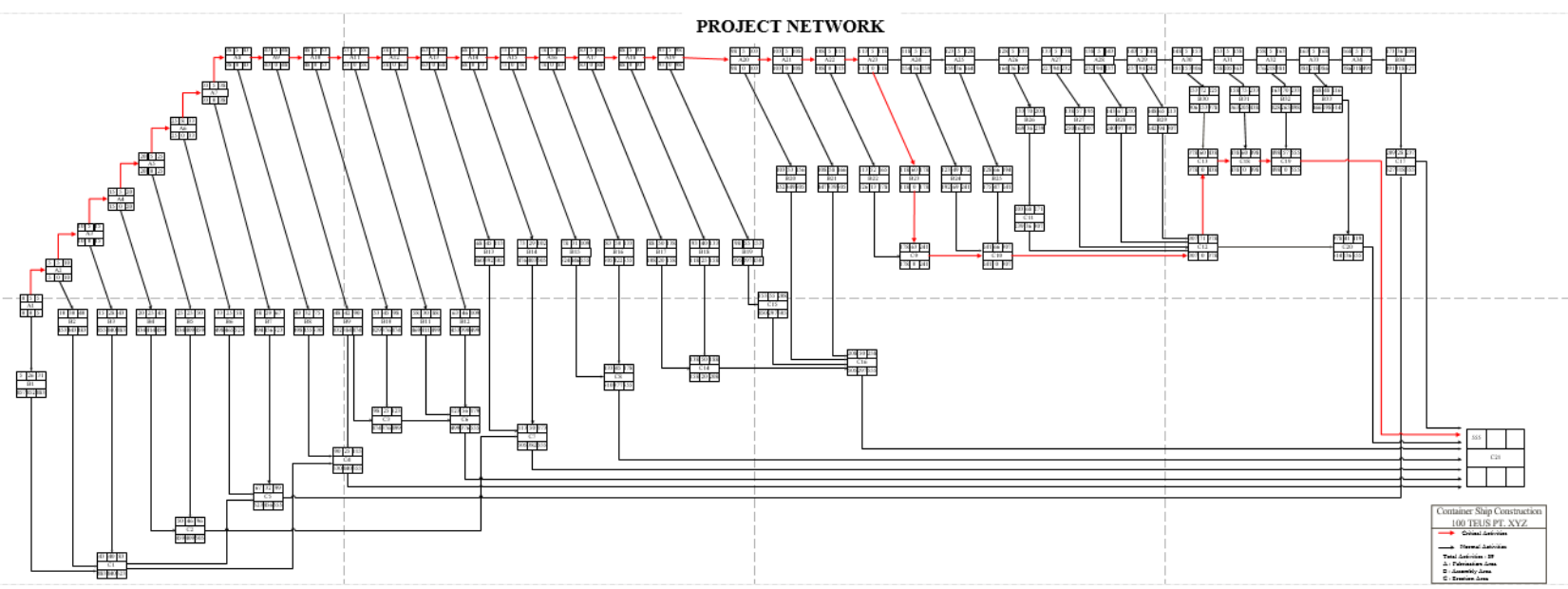

Fig 3. Container 100 TEUS Project Network

Then an analysis of the causes of delays in the project schedule is carried out, especially in the scope of critical activities. Based on the critical activity chain analysis, the activities that have a zero float value are:

\section{Conclusion}

From the results of research and discussions that have been carried out, several conclusions are obtained as follows:

1. By using the Critical Path Method CPM in scheduling, it is known that the age for a 100 TEUS ship construction project from start to finish is 555 days

2. Critical Path for a 100 TEUS ship construction project is an activity with the notation $\mathrm{A} 1, \mathrm{~A} 2$, A3, A4, A5, A6, A7, A8, A9, A10, A11, A12, A13, A14, A15, A16, A17, A18, A19, A20, A21, A22, A23, B23, C9, C10, C12, C13, C18, C19.

Suggestions from the results of research and discussion, it can be suggested to the contractor to:

1. Activities/works that are on a critical path can be given more attention because they can disrupt the overall 100 TEUS ship construction project.

2. If there is a delay in returning, the contractor can reschedule with methods of accelerating projects that are on the critical path.

However, this research was conducted at the planning stage of shipbuilding. So the analysis of this critical path method can be a risk prevention of time delays in shipbuilding projects. In addition, the
1. Fabrication: A1, A2, A3, A4, A5, A6, A7, A8, A9, A10, A11, A12, A13, A14, A15, A16, $\mathrm{A} 17, \mathrm{~A} 18, \mathrm{~A} 19, \mathrm{~A} 20, \mathrm{~A} 21, \mathrm{~A} 22, \mathrm{~A} 23$

2. Assembly: B23

3. Erection: C9, C10, C12, C13, C18, C19

analysis from this research can be used by company as a reference in contractual agreements with ship owners.

\section{References}

A. Harlan, F. B., \& Resda, D. P. (2019). Mitigation of Delay Risk in Ship Construction Project With Lean Approach. International Conference on Applied Economics and Social Science (pp. 160-164). Batam: Atlantis Press.

B. Imamah, M. P., \& Supriyanto, H. (2013). Evaluasi dan Perbaikan Proses Sub Assemblydengan Pendekatan Lean Risk di PT. PAL Indonesia (Persero). JURNAL TEKNIK ITS, 1-6.

C. Manalu, Z., \& Lestari, Y. D. (2015). PROJECT EFFECTIVENESS IMPROVEMENT: A CASE STUDY IN PT.X. JOURNAL OF BUSINESS AND MANAGEMENT, 587-593.

D. Moussourakis, J., \& Haksever, C. (2007). Models for Accurate Computation of Earliest and Latest Start Times and Optimal Compression in Project Networks. Journal of Construction Engineering and Management, 133(8), 600-608 
E. Newbold, R. C. (1998). Project Managament in the Fast Lane. CRC Press.

F. Pankaj, R, D., Kumar, A., \& Agarwal, R. (2020). Energy efficient path determination in wireless sensor network by critical path method. Malaya Journal of Matematik, 8(3), 797-802.

G. Shi, Q., \& Blomquist, T. (2012). A new approach for project scheduling using fuzzy dependency structure matrix. International Journal of Project Management, 30(4), 503 510.

H. Storch, R.L, Hammon, C.P, Bunch, H.M dan Moore, R.C. (1995). Ship Production 2nd Edition. Maryland: Cornell Maritime Press

I. Yamit. (2000). Manajemen Proyek Konstruksi (Edisi Ke-1).Yogyakarta (ID): Andi. 